\title{
Protrusion of the Rod Electrode in the Electrospinning Process
}

\author{
Jan Valtera, ${ }^{1}$ Lucie Vysloužilová, ${ }^{2}$ Jiří Komárek, ${ }^{1}$ Josef Skřivánek, ${ }^{1}$ Petr Žabka, ${ }^{1}$ \\ Jaroslav Beran, ${ }^{1}$ and David Lukáśs ${ }^{2}$ \\ ${ }^{1}$ Department of Textile Machine Design, Technical University of Liberec, Studentska 2, 46001 Liberec, Czech Republic \\ ${ }^{2}$ Department of Nonwovens and Nanofibrous Materials, Technical University of Liberec, Studentska 2, 46001 Liberec, Czech Republic
}

Correspondence should be addressed to Jan Valtera; jan.valtera@tul.cz

Received 24 July 2015; Accepted 27 September 2015

Academic Editor: Jeffery L. Coffer

Copyright (C) 2015 Jan Valtera et al. This is an open access article distributed under the Creative Commons Attribution License, which permits unrestricted use, distribution, and reproduction in any medium, provided the original work is properly cited.

\begin{abstract}
The paper focuses on the influence of the protrusion of the rod electrode on critical voltage in the DC electrospinning process. On the testing and industrial DC electrospinning devices, electrodes of any kind are extended towards the counter electrode. This provides the maximal, that is, supercritical, electric field intensity on the spinning-electrode orifice that is found to be higher than on the other supplementary parts. The principal study and experiments with basic apparatus were carried out and presented by Taylor in 1966. This study is focused on the arrangement closely related to the design of the real electrospinning device with respect to the safety and technological aspects. Results of the carried out experiments of the rod spinning-electrode are compared with the electrostatic simulation and analytical calculation. The presented effect of the electrode protrusion on the potential difference and the critical field strength introduces valuable information for the designers of electrospinning machines as well as for the setting up of the optimal technological parameters for producing modern nonwoven textile products.
\end{abstract}

\section{Introduction}

Nanofibers are mostly produced by the electrospinning. This technology has opened a wide range of industrial applications in many fields, for example, filtration [1], electrically conductive nanofibers [2], membranes for batteries [3], nanofiber composites [4], medicine and wound dressing [4], materials for tissue engineering [5], and materials for drug delivery system [6].

The principle of the electrospinning is based on a high voltage that is applied to an electrospinnable polymeric solution. The process begins with reaching of a specific value of the electric field strength $E_{c}$, which is called critical field intensity value, after overcoming of a liquid surface tension. For this purpose many technologies have been developed.

The basic technology for production of nanofibers is called the needle electrospinning. The main idea of this method is to dose a polymeric solution through a charged needle. Droplet of solution at the needle orifice is subjected to a strong electric field. After applying high voltage to the polymeric solution the Taylor cones formation can be observed [7] on the top of the droplet. The Taylor cone develops into a jet that produces fibers.

Another method was developed in 2003 by Yarin and Zussman [8]. This new technology is based on the use of a ferromagnetic suspension to electrospinning a polymer solution without needles. In the needleless way of electrospinning the principle of electrospinning from a free surface of polymeric layer is applied. In the environment of high external electric field the electrospun liquid is destabilized [9]. The effect of instability is described as Larmor-Tonks-Frenkel one. This effect has its nature in the self-organization by the mechanism of the "fastest forming instability," that is, of the fastest growing capillary wave [10-12]. Plenty of polymer jets are created by using needleless spinning-electrodes. These jets are made from a free liquid surface of a thin polymeric layer. The advantage of this method is, in terms of productivity of nanofibers, easier maintenance of a spinning-electrode and more homogeneous electric field. In 2004 the first machine for the mass production was developed and introduced for commercialization under the brand name Nanospider [13]. 
Apart from the previously mentioned technologies that use the DC voltage, the researchers at the Technical University of Liberec introduced a new method, where the AC voltage is applied [14]. This technology brings new possibilities as only one electrode is required.

All the presented technologies are based on the use of high voltage. From the device designing and construction point of view, it is crucial to maintain both the sufficient power supply to run the electrospinning process and also the sufficient electrical isolation of devices at the critical field intensities. The electrode design and its connection to the frame with zero potential bring heavy demands on the device construction and need to be therefore investigated. Exact investigation of the electrode protrusion length on the critical voltage difference between the spinning-electrode and a collector would therefore contribute to optimal arrangement of electrospinning devices. Thus, device size can be optimized, while maintaining equivalent production and safety parameters.

\section{Configuration of Electrodes in Electrospinning Process}

The configuration of DC based electrospinning technology is shown in the scheme in Figure 1. This scheme describes an example of vertical configuration where the fibers are transported from the positively charged spinning-electrode towards the negatively charged counter electrode, that is, collector. Both electrodes are electrically supplied and are connected by means of isolated parts to the device frame that is connected to the zero potential. There are many types of configurations and the rod electrodes used for the electrospinning process [16].

The formation of the Taylor cone from a free polymeric layer is shown in Figure 2. The critical electrical intensity $E_{c}$ required to form the Taylor cone on the free liquid surface can be calculated as

$$
E_{c}=\sqrt[4]{\frac{4 \gamma \rho g}{\varepsilon^{2}}}
$$

where $\gamma$ represents the liquid surface tension, $\rho$ the liquid density, $g$ the gravitational acceleration, and $\varepsilon$ the air permittivity [9].

In order to maintain the required value of $E_{c}$, a certain value of potential difference at the electrodes needs to be set. Depending on the electrode geometry, material, and also the geometry of additional parts in the electrospinning setup, the necessary potential difference can vary. The influence of protrusion of rod electrode in the electrospinning setup was studied and presented by Taylor in 1966 [17]. In this work Taylor modified Zeleny's single-plate apparatus [18] and tested the "two-plate" one that is shown in Figure 3. Based on the experiment results [15] Taylor formulated (2) that expresses the electrical force $P$ as a function of the voltage $V$, the distance between plates $H$, the protrusion of the rod electrode $L$, and the outer radius $R$ :

$$
P=\frac{V^{2} L^{2}}{4 H^{2}} \cdot \frac{1}{\ln (2 L / R)-3 / 2} .
$$

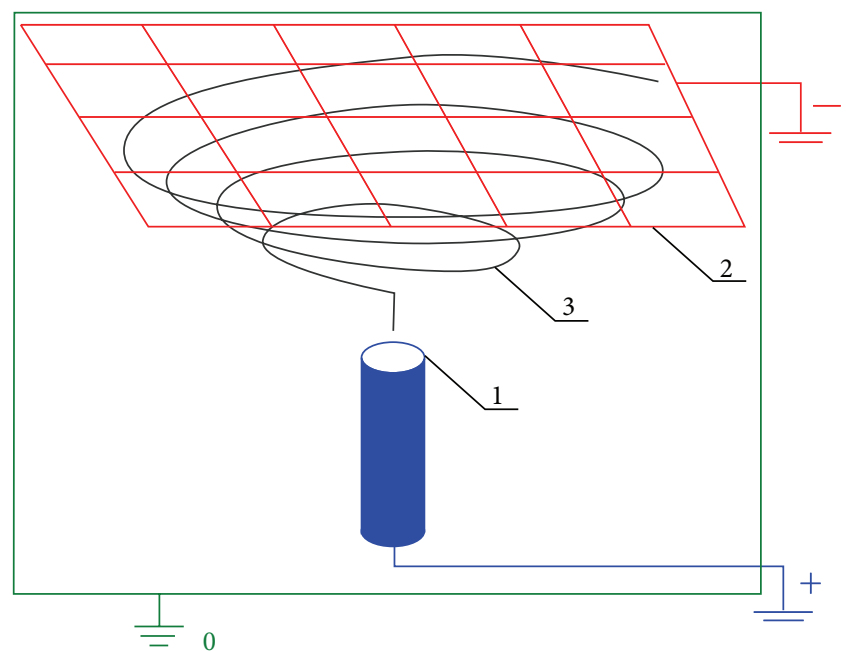

FIGURE 1: Vertical configuration of the DC based electrospinning setup: the nanofibers (3) created at the positively charged electrode (1) are collected on the oppositely charged electrode (2) inside a device frame with zero potential.

Considering the critical condition of the electrospinning process, the electrical force $P$ is equal to the capillary force $P_{c}$ at the perimeter of the Taylor cone with the vertical angle $\gamma$ (as shown in Figure 2(c)). Consider

$$
P_{c}=2 \pi R T \cdot \cos \left(\frac{\gamma}{2}\right)=\pi R T \cdot 1.3,
$$

where $T$ represents the symbol for the capillary tension used by Taylor. At the critical stage therefore

$$
\pi R T \cdot 1.3=\frac{V_{c}^{2} L^{2}}{4 H^{2}} \cdot \frac{1}{\ln (2 L / R)-3 / 2},
$$

and the critical voltage is then

$$
V_{c}^{2}=\frac{4 H^{2}}{L^{2}}\left(\ln \frac{2 L}{R}-\frac{3}{2}\right) \cdot \pi R T \cdot 1.3 \cdot 0.09 .
$$

This formulation is presented in the work [15]. Here the distance between plates $H$, the protrusion of the rod electrode $L$, and the outer radius $R$ are expressed in units $\mathrm{cm}$, capillary tension $T$ is expressed in $\mathrm{mN} / \mathrm{m}$, and $V_{c}$ is expressed in $\mathrm{kV}$ due to the unit correction factor 0.09 .

\section{Experiment}

The aim of this experiment is to determine the optimal protrusion of the rod electrode above a plate and examine the influence of this plate on the start of the DC based electrospinning process. With respect to Taylor's analysis, the experiment with axisymmetric geometry of electrodes and plate was set up. This experimental setup, however, reflects the spinning devices, where nonconductive materials may be used. Therefore, the material of the lower plate is made of polycarbonate (Makroclear). The scheme of our experimental apparatus is shown in Figure 4. Collector 1 (the negatively 


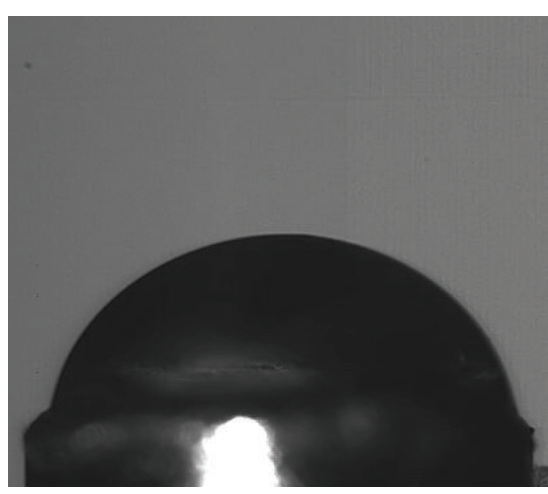

(a)

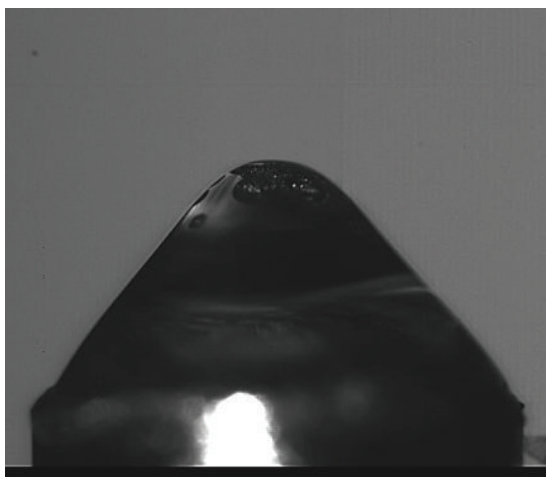

(d)

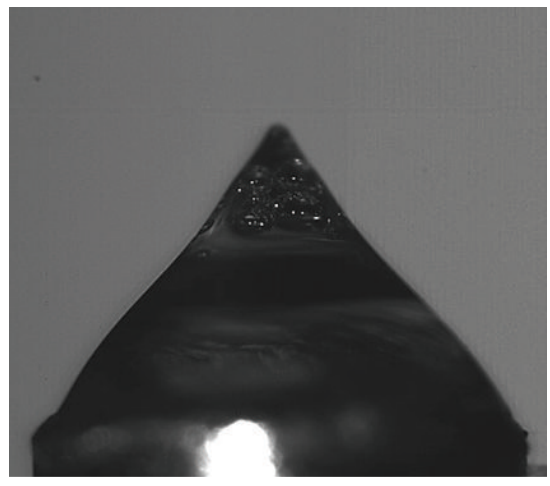

(g)

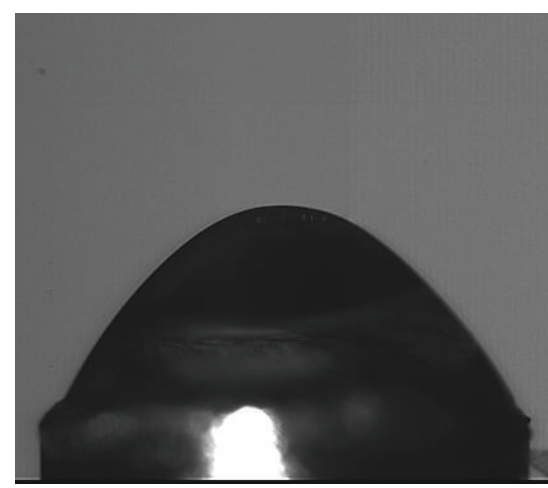

(b)

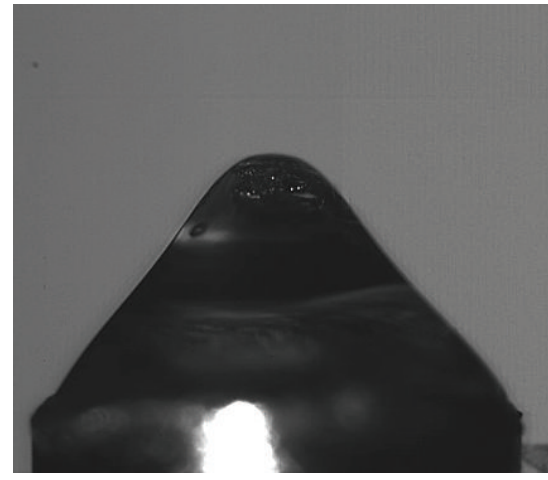

(e)

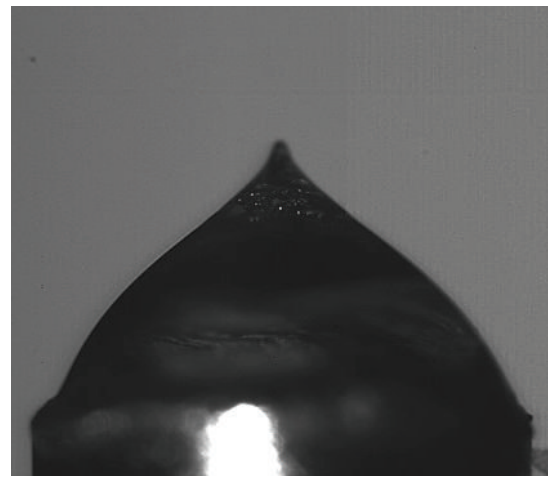

(h)

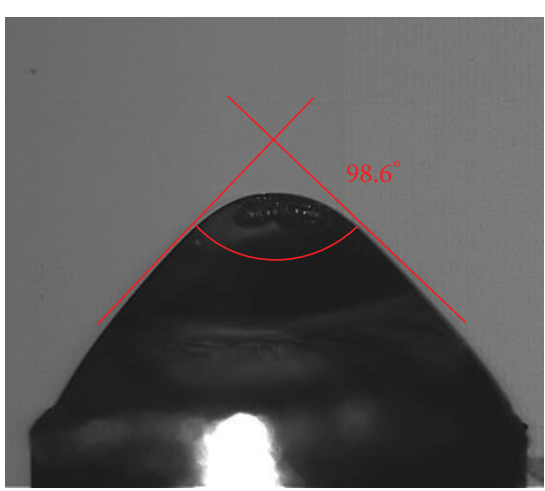

(c)

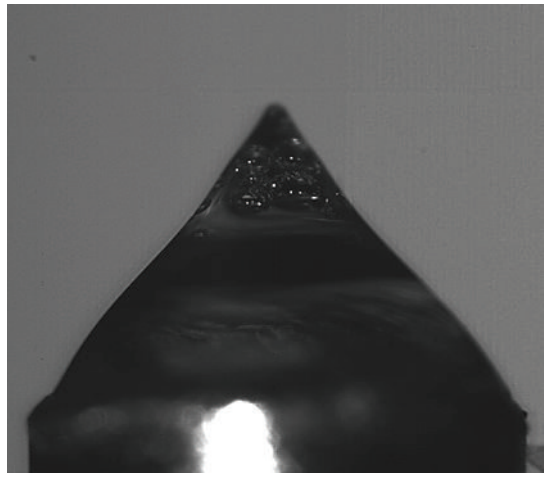

(f)

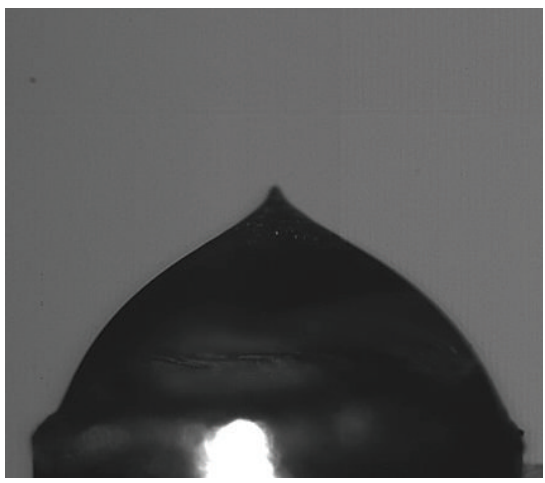

(i)

Figure 2: The Taylor cone formation from the free polymeric layer and the start of the electrospinning process: Taylor cone formation (a-c) and relaxation of polymeric droplet ( $\mathrm{d}-\mathrm{i})$. Start of the spinning process (i). Reaction of electric and capillary forces can be observed. The angle of the Taylor cone $\gamma(\mathrm{c})$. Needle electrospinning of $12 \mathrm{wt}$.\% PVA, $U=30 \mathrm{kV}$. Electrospinning was carried out at laboratory conditions.

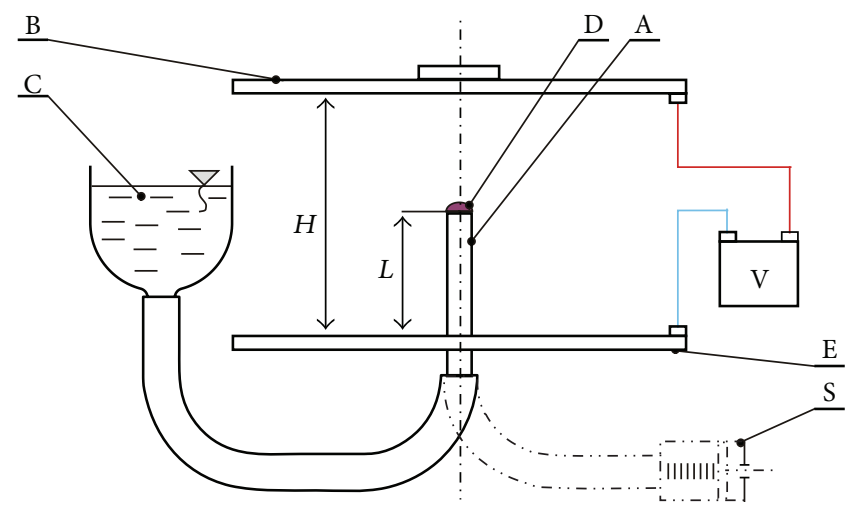

FIgure 3: The scheme of Taylor's two-plate apparatus [15]: A, metal tube electrode; B, counter plate electrode; C, polymer supplied from a reservoir through flexible tube; $\mathrm{D}$, polymeric droplet; $\mathrm{E}$, plate; $\mathrm{S}$, polymer supplied by a graduated syringe (alternative option for measuring the rate or delivery). 


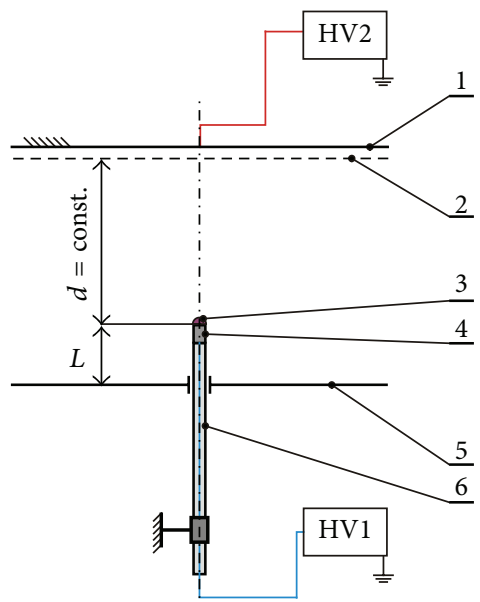

(a)

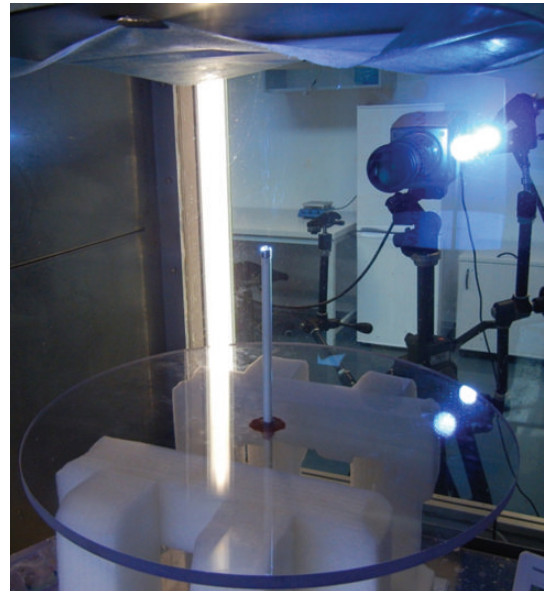

(b)

FIGURE 4: (a) Scheme of the experimental setup and (b) picture of experimental apparatus.

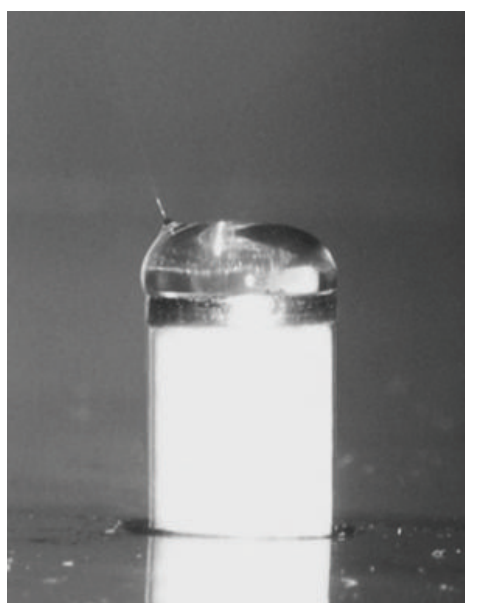

(a)

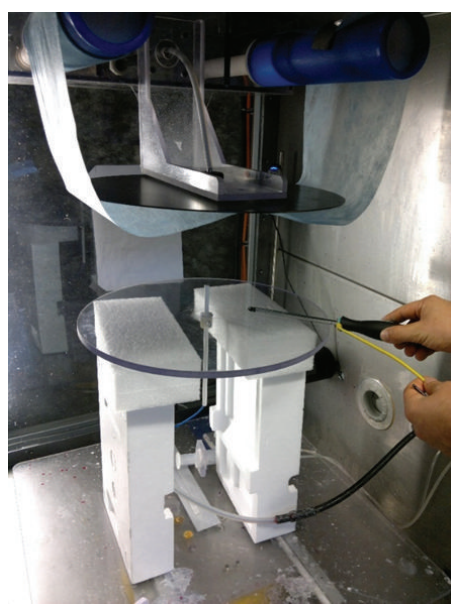

(b)

Figure 5: (a) Polymeric droplet with a jet under the critical potential and (b) removing of the remaining charge after the experiment.

charged electrode) is a steel plate with outer diameter of $400 \mathrm{~mm}$ and thickness of $2 \mathrm{~mm}$. The nonwoven textile spunbond (Pegas Nonwovens, CZ) 2 textile was placed on the collector from the bottom side to collect fibres. Rod electrode 6 was made of hollow plastic rod with the outer diameter of $7.6 \mathrm{~mm}$. The tip of rod 6 was covered by steel cylinder 4 with outer diameter of $7.6 \mathrm{~mm}$ and thickness of $1 \mathrm{~mm}$. The high voltage was transmitted to cylinder 4 by means of a cable through the inner hole of plastic rod 6. Plate 5 was made of PMMA with outer diameter of $400 \mathrm{~mm}$ and thickness of $10 \mathrm{~mm}$. The length between collector 1 and electrode 4 (6) $d$ remained constant at $150 \mathrm{~mm}$ during the whole experiment. In order to place polymeric droplet 3 of a certain volume on the tip of electrode 4 , the precise syringe was used. During the experiment, protrusion $L$ of rod 6 electrode above plate 5 was changed.
Plate 5 was expected to be exposed to the electrical field and therefore influence the electrical field strength at electrode orifice 4 . The required potential difference to polymeric droplet 3 under various rod protrusions $L$ is therefore the main subject of this experiment. The critical potential was assessed when the first jet occurred on the polymeric droplet, as it is shown in Figure 5(a). The actual value of the voltage potential was determined from the high voltage power supply.

The room temperature was $24^{\circ} \mathrm{C}$ and $\mathrm{RH}$ was $51 \%$. For the tested polymeric material, the polyvinyl alcohol (PVA, Sloviol R16) (from Chemicke zavody Novaky, SK) was used. For our experiment, a solution of $12 \mathrm{wt} . \%$ PVA in distilled water was prepared. The capillary tension of the polymer is $40 \mathrm{mN} / \mathrm{m}$.

The experiment was carried out for the 6 values of electrode protrusion with the following steps: 


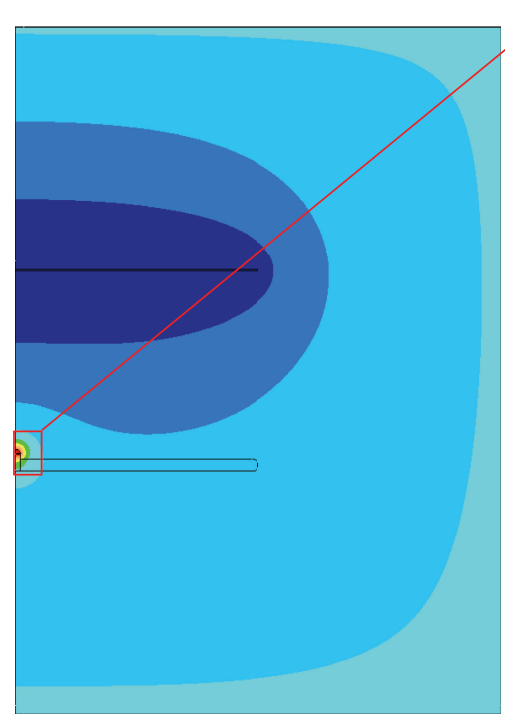

(a)

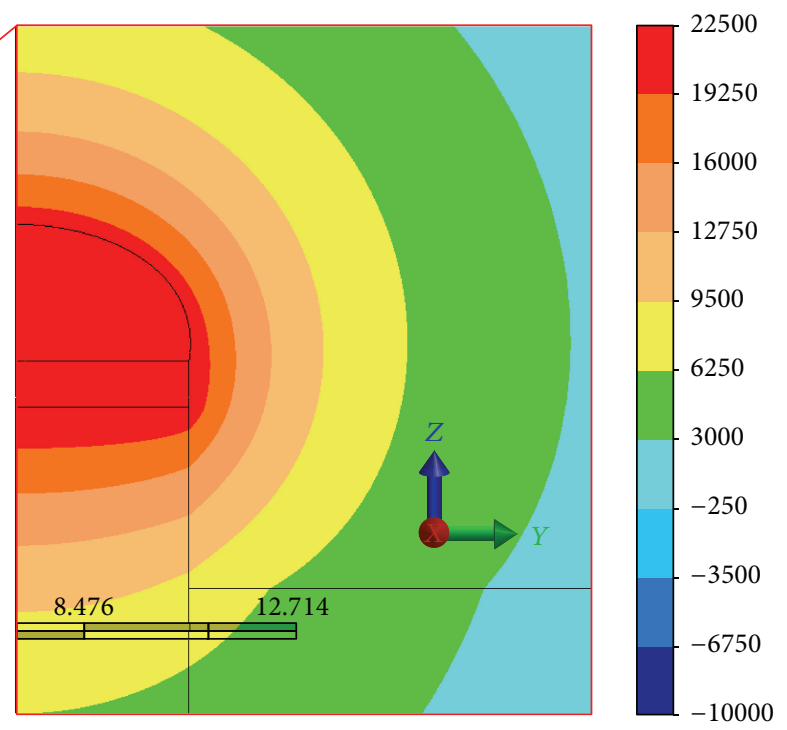

(b)

FIGURE 6: Analysis results: (a) graphical distribution of potential and (b) detailed view on the tip of the rod electrode.

(1) The required distance $L$ was set accordingly.

(2) The droplet of volume $0.1 \pm 0.05 \mathrm{~mL}$ was injected on the tip of the electrode by a syringe.

(3) The voltage differential was switched on for $10 \mathrm{sec}-$ onds.

(4) The voltage was switched off for 60 seconds and the plate surface was discharged by touching of steel bar that was connected to the ground potential, as shown in Figure 5(b). The remaining polymeric droplet was removed from the electrode.

(5) The set value of the voltage difference has been increased by repeating steps (3) and (4), until the critical value was reached. The precise value of the critical voltage was measured by means of several tests with slightly increased and decreased voltage.

By means of this procedure, the critical voltage for 6 variants of the electrode protrusion was determined and the results are shown in Table 1.

\section{Electrostatic Analysis}

The aim of the simulation of the presented task was to verify the assumption of constant critical intensity on the polymeric droplet at the moment of reaching the critical voltage. Based on the previous analysis of electrostatic problems of electrodes with different geometry, described in [19], the software Autodesk Simulation Multiphysics was used. It is an advanced software enabling calculation of engineering tasks by means of the finite element method (FEM).

Thanks to the axial symmetry of the studied problem, it can be solved as a 2-dimensional problem with axial
TABLE 1: Measured values of critical voltage.

\begin{tabular}{lcccccc}
\hline Protrusion $L[\mathrm{~mm}]$ & 2.5 & 5 & 10 & 20 & 50 & 100 \\
\hline Electrode potential $[\mathrm{kV}]$ & 24 & 22.5 & 14 & 9.2 & 8.8 & 8.5 \\
Collector potential $[\mathrm{kV}]$ & 10 & 10 & 10 & 9 & 8 & 8 \\
Potential difference $[\mathrm{kV}]$ & 34.5 & 32.5 & 24 & 18.2 & 16.8 & 16.5 \\
Upper variance limit $[\mathrm{kV}]$ & 37 & 34 & 25 & 18.5 & 17 & 16.6 \\
Lower variance limit $[\mathrm{kV}]$ & 32 & 31 & 23 & 18 & 16.7 & 16.4 \\
\hline
\end{tabular}

symmetry. In order to set the FEM model, the 2D geometry was created according to dimensions described in the experiment. The material properties of tested parts were defined in the model. The applied voltage of the electrodes was set accordingly. The outer space, that is, the simulation box, was defined as a cylinder with outer diameter of $800 \mathrm{~mm}$ and applied voltage of $0 \mathrm{~V}$ on its outer boundary. In order to perform the simulation closely related to the experiment, the polymeric droplet was incorporated into the model. The geometry of the polymeric droplet was defined by means of spline corresponding to the droplet image and also to the overall volume of $0.1 \mathrm{~mL}$.

For the simulation, the Electrostatic Field Strength and Voltage type analysis was selected. Figure 6(a) illustrates the voltage, that is, electric potential, distribution over the model and Figure 6(b) shows detailed view on the tip of the electrode where the required voltage is applied. Figure $7(\mathrm{a})$ illustrates the electric field strength distribution with the detailed view shown in Figure 7(b).

In Figure $7(\mathrm{~b})$ the point of maximum intensity on the polymeric droplet can be observed. Because of changing curvature of the oval polymeric shape, the maximum value on the polymeric droplet is close to its outer diameter. 


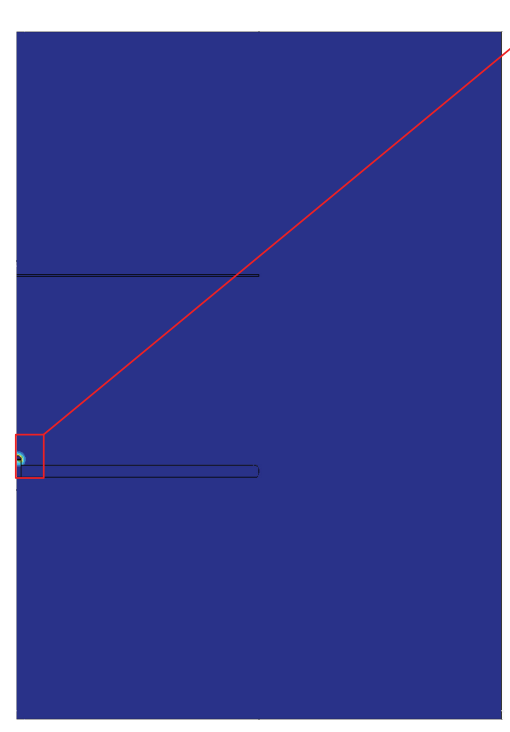

(a)

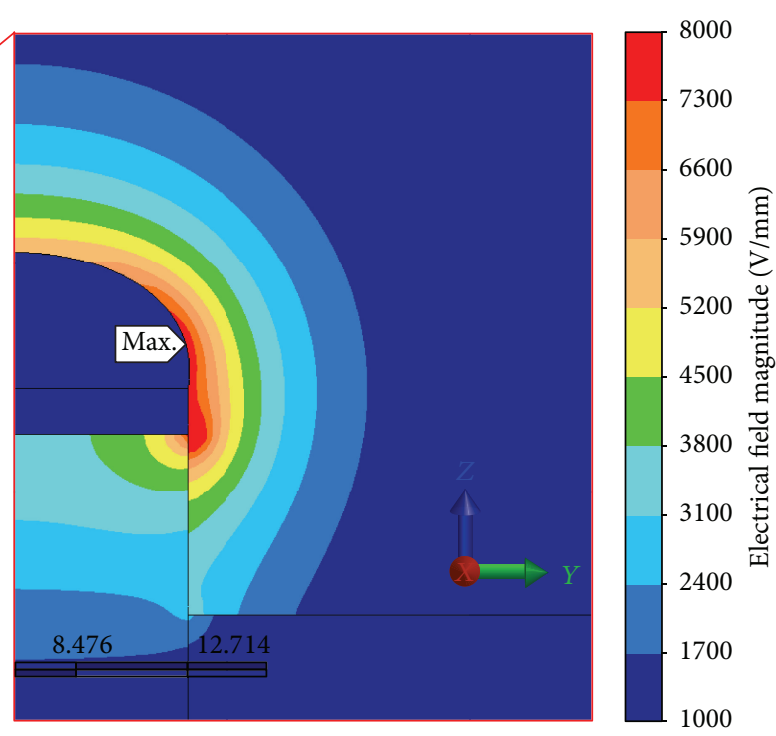

(b)

FIGURE 7: (a) Spatial distribution of electrical field strength and (b) detailed view on the tip of the rod electrode.

\section{Results and Discussion}

Results from the experiment are shown in Table 1. The dependence of the electrical potential based on the protrusion is shown in Figure 8. In this figure, the experimental results are compared with analytical calculation (5) that can be expressed for the carried out experiment as

$$
V_{c}=2 \frac{L+D}{L} \cdot \sqrt{\ln \frac{2 L}{0.38}-\frac{3}{2}} \cdot \sqrt{\pi \cdot 0.38 \cdot 40 \cdot 1.3} \cdot 0.3 .
$$

From the presented results we can observe the similar progressive character of both the experimental and Taylor's analytical courses. In both cases the critical voltage $V_{c}$ increases while the electrode protrusion is reduced. In the case of nonconductive materials of the rod and the plate in the experimental setup, there is only marginal change of critical voltage $V_{c}$ from approximately $16 \mathrm{kV}$ on the electrode protrusion of $100 \div 20 \mathrm{~mm}$. The progressive increase of critical potential difference is present at the protrusion value of $20 \mathrm{~mm}$, where the critical voltage begins to increase rapidly up to $34.5 \mathrm{kV}$ at the minimal measured electrode protrusion of $2.5 \mathrm{~mm}$. It means that electrodes with higher protrusion are not more efficient as they increase the required inner space without significant impact on the critical voltage reduction. In the case of the analytical calculation of Taylor's setup (built up from conductive materials), the higher values of critical voltage were determined. Figure 9 illustrates results of the critical electric intensity $E_{c}$ with respect to the electrode protrusion. $E_{c}$ was determined by electrostatic analysis using the FEM method. In this analysis, the critical states of electrospinning were described by applied voltage corresponding to the experimentally determined values of critical voltage $V_{c}$. The maximal values of electric intensity on the polymeric droplet were then evaluated and compared with the analytical calculation based on formula (1). The analysis results clearly show a progressive increase within the protrusion of $20 \mathrm{~mm}$.

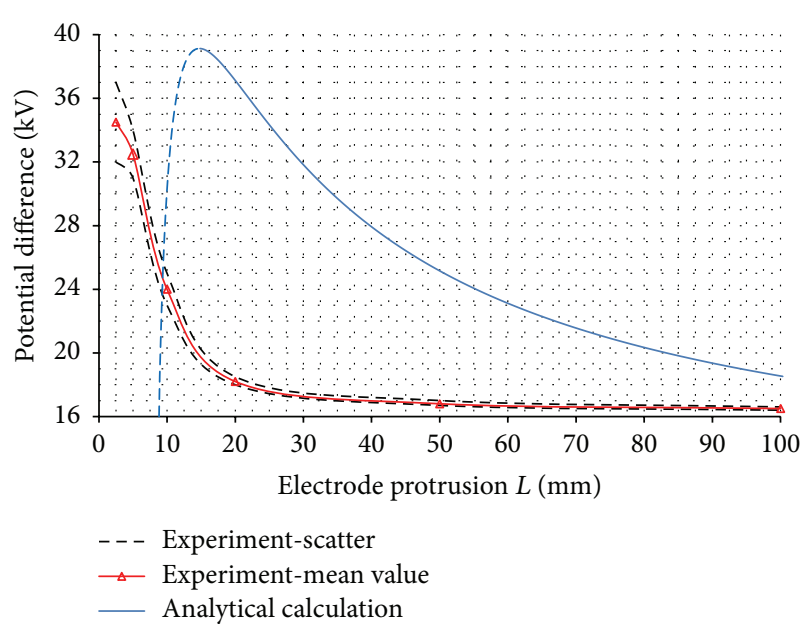

FIGURE 8: Dependence of the potential difference with respect to the rod electrode protrusion. Experimental results are compared with the theoretical prediction.

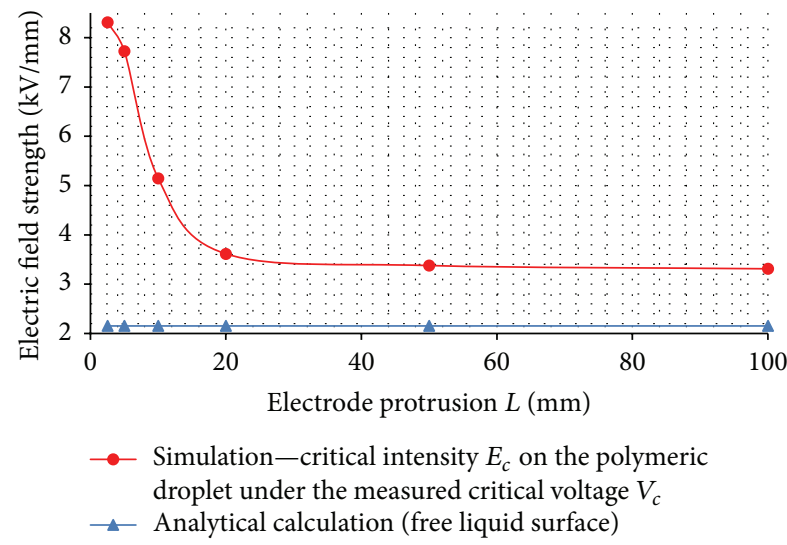

FIGURE 9: Dependence of the critical electric field strength on the rod electrode protrusion. 
In comparison, the analytical calculation defines the critical value only by polymer based factors and therefore remains constant. However, at the maximal electrode protrusion, the difference between results of analytical calculation and simulation is about $30 \%$.

\section{Conclusion}

Presented work focuses on the influence of the rod electrode protruded above a plate at the critical condition of the electrospinning process. The aim of this work was to clarify new approaches to obtain optimal arrangement for electrospinning devices and thus optimize the electrospinning technology. Results of carried out experiments with the electrode and the plate made of nonconductive materials were compared with results of the corresponding setup made of conductive materials (based on Taylor's experiments). This proves that, in the case of using nonconductive materials, the lower critical voltage $V_{c}$ is needed for starting the electrospinning process. This has positive impact on the operating conditions as even the lower critical voltage can satisfy the required condition for the electrospinning process. The lower operating voltage reduces the requirements on the electrospinning device size from the electrical and also the safety point of view.

Another aspect of the presented study is related to the optimal protrusion of the rod electrode. According to the experimental results, electrode protrusion from 20 to $100 \mathrm{~mm}$ does not bring any significant decrease in the critical voltage. For the tested setup the optimal protrusion is approximately $20 \mathrm{~mm}$. This brings positive impact on the whole design of the device as it reduces the inner space required for efficient and safe operation of the device.

The devised FEM model enables one to analyze the electrical field strength distribution on the polymeric droplet. However, characteristic of the critical field strength course at the electrode orifice does not meet the assumption given by the theoretical calculation for a free liquid surface. This may be caused by an inappropriate shape of the polymeric droplet that goes through several stages, while Taylor's cone is developing. It is assumed that, in spite of only 10 s long test, the surface of the plate was affected by certain amount of electrical charge. Furthermore, the level of plate charging during the tests was more significant as the electrode protrusion was lowered. Therefore, the electric field strength, based on the measured voltage, does not describe the state appropriately. In order to confirm these assumptions further experiments and electrostatic analysis with the droplet corresponding to different stages of Taylor's cone formation should be undertaken.

\section{Conflict of Interests}

The authors declare that there is no conflict of interests regarding the publication of this paper.

\section{Acknowledgments}

This paper was written at the Technical University of Liberec as part of the Research and Development of Devices for Production of Nanofibers project with the support of the Specific University Research Grant, as provided by the Ministry of Education, Youth and Sports of the Czech Republic in the year 2015. The authors thank the Ministry of Education, Youth and Sports of the Czech Republic for the financial support from the OPR \& DI project Centre for Nanomaterials, Advanced Technologies and Innovation (CZ.1.05/2.1.00/01.0005). The authors also thank the Nanoprogress z.s.p.o. for providing its laboratory for carried out experiments.

\section{References}

[1] S. Rangarajan, K. Mehta, and G. G. Chase, "Nanofibers in coalescer filter media," in Proceedings of the Fall Topical Conference of the American Filtration and Separations Society, pp. 177-185, 1999.

[2] A. G. MacDiarmid, W. E. Jones Jr., I. D. Norris et al., "Electrostatically-generated nanofibers of electronic polymers," Synthetic Metals, vol. 119, no. 1-3, pp. 27-30, 2001.

[3] I. D. Norris, M. M. Shaker, F. K. Ko, and A. G. Macdiarmid, "Electrostatic fabrication of ultrafine conducting fibers: polyaniline/polyethylene oxide blends," Synthetic Metals, vol. 114, no. 2, pp. 109-114, 2000.

[4] H.-J. Jin, S. V. Fridrikh, G. C. Rutledge, and D. L. Kaplan, "Electrospinning Bombyx mori silk with poly (ethylene oxide)," Biomacromolecules, vol. 3, no. 6, pp. 1233-1239, 2002.

[5] D. Li and Y. Xia, "Electrospinning of nanofibers: reinventing the wheel?" Advanced Materials, vol. 16, no. 14, pp. 1151-1170, 2004.

[6] E.-R. Kenawy, G. L. Bowlin, K. Mansfield et al., "Release of tetracycline hydrochloride from electrospun poly(ethyleneco-vinylacetate), poly(lactic acid), and a blend," Journal of Controlled Release, vol. 81, no. 1-2, pp. 57-64, 2002.

[7] G. Taylor, "Disintegration of water drops in an electric field," Proceedings of the Royal Society of London Series A: Mathematical and Physical Sciences, vol. 280, no. 1382, pp. 383-397, 1964.

[8] A. L. Yarin and E. Zussman, "Upward needleless electrospinning of multiple nanofibers," Polymer, vol. 45, no. 9, pp. 29772980, 2004.

[9] D. Lukas, A. Sarkar, and P. Pokorny, "Self-organization of jets in electrospinning from free liquid surface: a generalized approach," Journal of Applied Physics, vol. 103, no. 8, Article ID 084309, 2008.

[10] J. Frenkel, Kinetic Theory of Liquids, Reissue Edition, Dover Publications, New York, NY, USA, 1955.

[11] L. Tonks, "A theory of liquid surface rupture by a uniform electric field," Physical Review, vol. 48, no. 6, pp. 562-568, 1935.

[12] J. Larmor, "On the influence of electrification on ripples," Proceedings of the Cambridge Philosophical Society. Mathematical and Physical Sciences, vol. 7, pp. 69-72, 1890.

[13] O. Jirsak, F. Sanetrnik, D. Lukas, V. Kotek, L. Martinova, and J. Chaloupek, "A method of nanofibers production from a polymer solution using electrostatic spinning and a device for carrying out the method," Patent, WO2005024101, 2005.

[14] P. Pokorny, E. Kostakova, F. Sanetrnik et al., "Effective AC needleless and collectorless electrospinning for yarn production," Physical Chemistry Chemical Physics, vol. 16, no. 48, pp. 26816-26822, 2014. 
[15] G. Taylor and M. D. Van Dyke, "Electrically driven jets," Proceedings of the Royal Society of London A, vol. 313, no. 1515, pp. 453-475, 1969.

[16] K. Pejchar, L. Vyslouzilova, J. Beran, D. Lukas, M. Bilek, and P. Pokorny, "The slit needleless electrode for the electrospinning," in Proceedings of the 5th International Conference of Nanocon, Brno, Czech Republic, October 2013.

[17] G. Taylor, "Oblique impact of a jet on a plane surface," Philosophical Transactions of the Royal Society A: Mathematical Physical and Engineering Sciences, vol. 260, no. 1110, 1966.

[18] J. Zeleny, "The electrical discharge from liquid points, and a hydrostatic method of measuring the electric intensity at their surfaces," Physical Review, vol. 3, no. 2, pp. 69-91, 1914.

[19] L. Vyslouzilova, J. Valtera, K. Pejchar, J. Beran, and D. Lukas, "Design of coaxial needleless electrospinning electrode with respect to the distribution of electric field," Applied Mechanics \& Materials, vol. 693, pp. 394-399, 2014. 

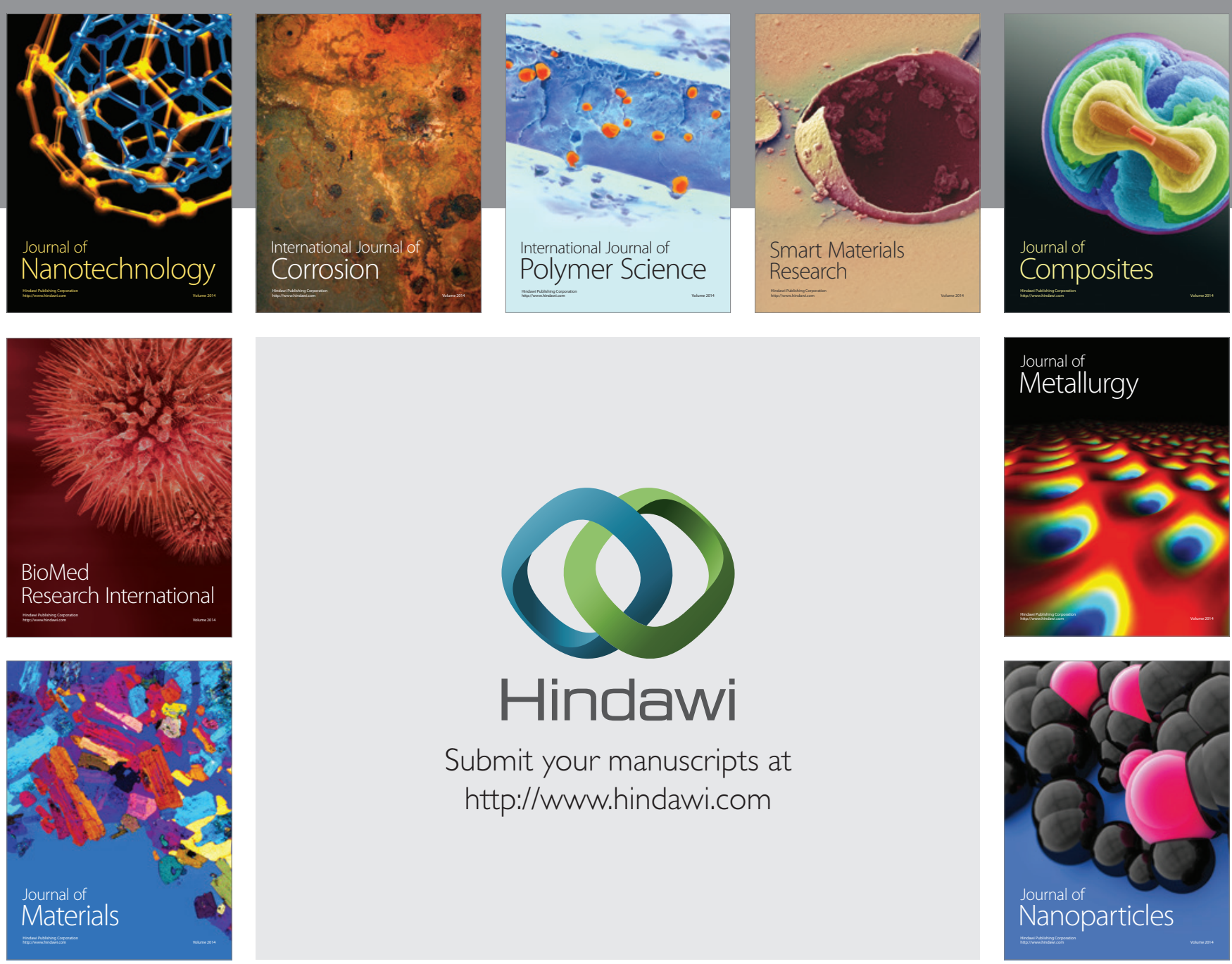

Submit your manuscripts at http://www.hindawi.com
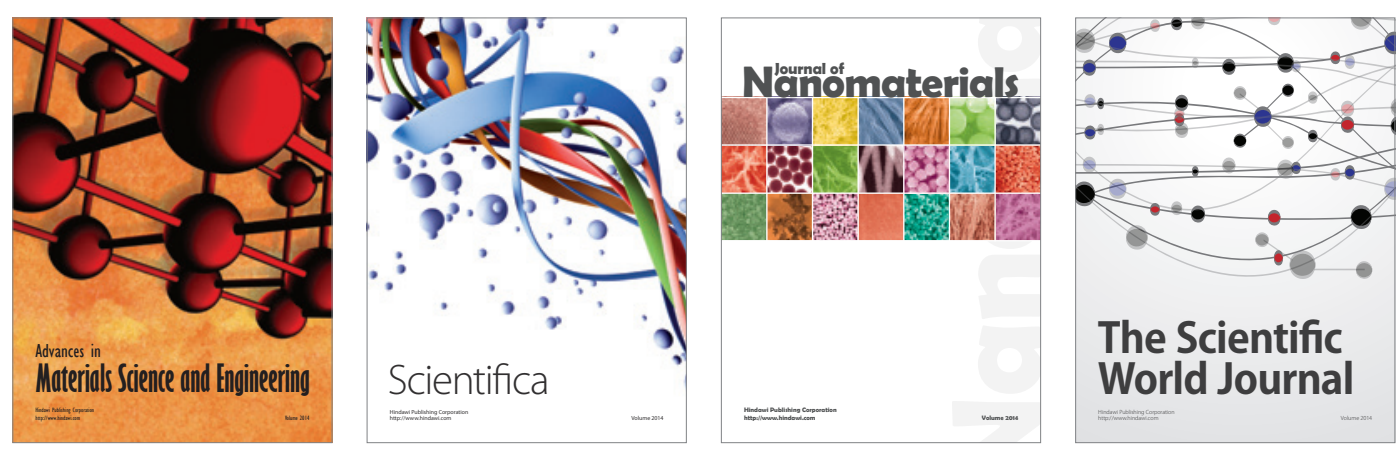

\section{The Scientific World Journal}
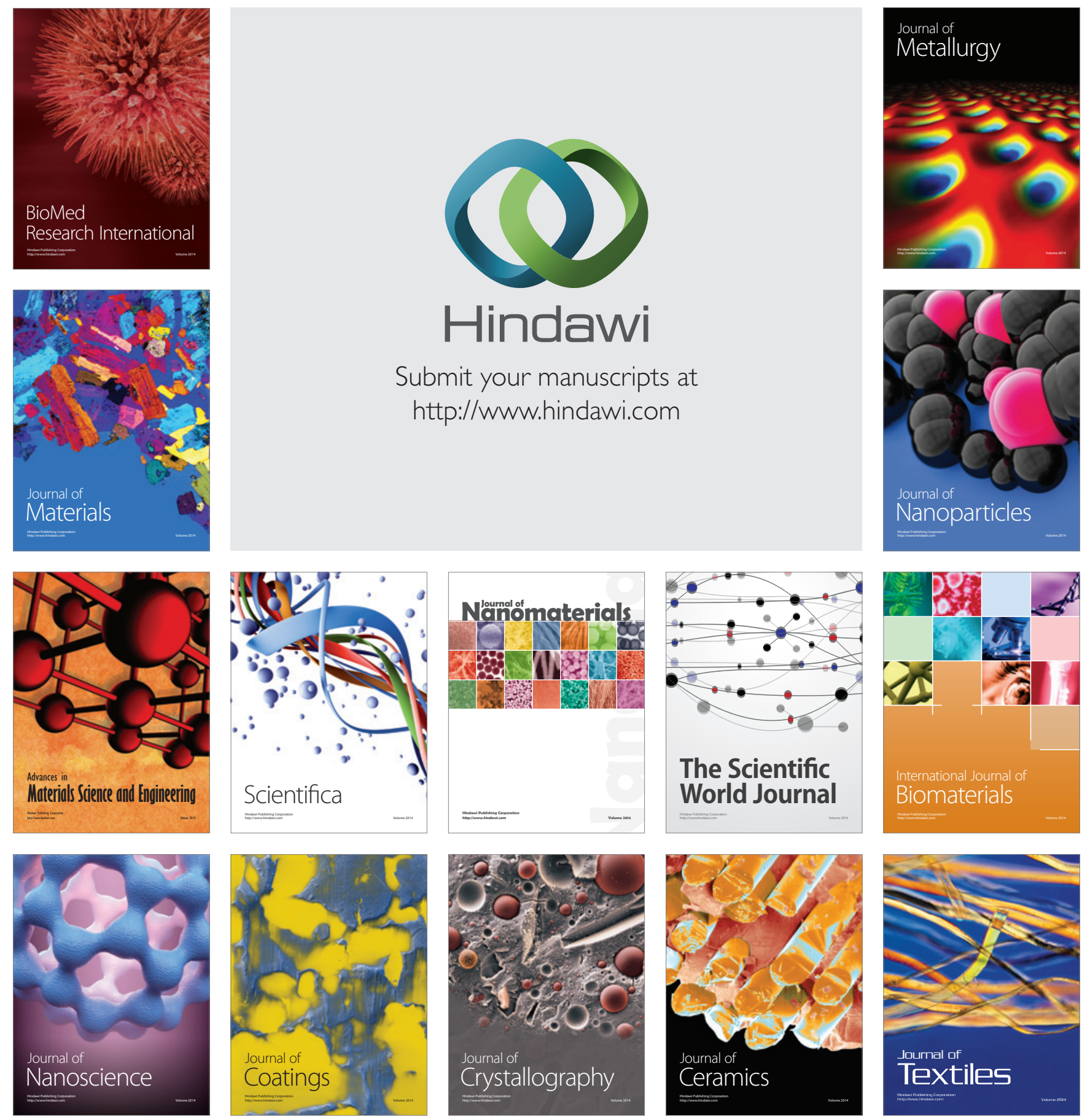